\title{
Differential distributions for single top quark production at the $\mathrm{LHeC}$
}

\author{
Meisen Gao $\oplus^{1, *}$ and Jun Gao $\circledast^{1,2,3, \dagger}$ \\ ${ }^{1}$ INPAC, Shanghai Key Laboratory for Particle Physics and Cosmology, School of Physics and Astronomy, \\ Shanghai Jiao-Tong University, Shanghai 200240, China \\ ${ }^{2}$ Key Laboratory for Particle Astrophysics and Cosmology (MOE), Shanghai 200240, China \\ ${ }^{3}$ Center for High Energy Physics, Peking University, Beijing 100871, China
}

(Received 8 April 2021; accepted 17 August 2021; published 15 September 2021)

\begin{abstract}
We present a phenomenological study of the single top (anti)quark production with leptonic decays at the Large Hadron Electron Collider at next-to-leading order (NLO) in QCD. We focus on various differential distributions in a fiducial region. The NLO corrections can reduce the fiducial cross section by $14 \%$. We find that the NLO predictions exhibit strong stability under scale variations for most observables considered, while the scale variations at leading order dominate in the theoretical uncertainties. We propose a method of determining the top quark mass using the measurement of the average transverse momentum of the charged lepton. The scale variations at the NLO induce a theoretical uncertainty of about $1.3 \mathrm{GeV}$ of the extracted top quark mass. The statistical error of the extracted top quark mass amounts to $1.1 \mathrm{GeV}$. We also investigate the impact of the QCD corrections and the scale variations in searches for the anomalous $W t b$ couplings.
\end{abstract}

DOI: 10.1103/PhysRevD.104.053005

\section{INTRODUCTION}

The Large Hadron Electron Collider ( $\mathrm{LHeC})[1-3]$ is a proposed facility for using a newly built electron beam of $60 \mathrm{GeV}$ or higher energy to collide with the intense hadron beams of the LHC. As the high luminosity phase of the LHC will accumulate a total integrated luminosity of $3000 \mathrm{fb}^{-1}$, the $\mathrm{LHeC}$ is expected to reach a total integrated luminosity of $100 \mathrm{fb}^{-1}$. Such a program will be devoted to probing the energy frontier and complementing the discovery potential of the LHC with measurements of deep inelastic scattering (DIS). It can be used to study the parton structure of the proton and QCD dynamics [4-10], Higgs physics [11-26], trilinear couplings of gauge bosons $[27,28]$, top quark physics [29-39], and new resonances [40,41].

In particular, the $\mathrm{LHeC}$ will provide a cleaner environment for the study of single top production [31]. At the LHC, assuming a top quark mass of $172.5 \mathrm{GeV}$, the top quark pair and single top production cross sections are $\sigma_{t \bar{t}}=984.5 \mathrm{pb}$ and $\sigma_{t+\bar{t}}=245 \mathrm{pb}$ at $\sqrt{s}=14 \mathrm{TeV}[42-$ 44]. The processes of single top production contain $s, t$, and $W t$ channels which are all related to the Wtb vertex.

\footnotetext{
*gmason@sjtu.edu.cn

jung49@sjtu.edu.cn
}

Published by the American Physical Society under the terms of the Creative Commons Attribution 4.0 International license. Further distribution of this work must maintain attribution to the author(s) and the published article's title, journal citation, and DOI. Funded by SCOAP .
Theoretical efforts have been devoted to improving the theoretical predictions [45-83]. Single top quark production has been used to measure the Cabibbo-KobayashiMaskawa matrix element $V t b[84,85]$ and to extract the top quark mass [86-88]. In addition, various new physics [8999] have been searched for at the LHC, with no discovery yet. For the $\mathrm{LHeC}$, single top quark production via chargedcurrent DIS is dominant in all the top quark production channels. We can utilize this unprecedented facility to measure the precise properties of the top quark and search for new physics.

In this work we present a fully differential calculation of the single top quark production at the $\mathrm{LHeC}$ in the fiveflavor scheme (5FS) at next-to-leading order (NLO) in QCD. We focus on the leptonic decays of the top quark. The hadronic decays will be affected more by various nonperturbative effects of QCD and also the standard model (SM) backgrounds. We include full off-shell and nonresonant contributions in our calculation. We study the QCD corrections to various differential distributions in a typical fiducial region of the LHeC. Specifically, we show their impact on the precision measurement of the top quark mass and the searches of the anomalous Wtb couplings.

The rest of our paper is organized as follows. In Sec. II, we present our theoretical framework and the numerical results of the total cross sections. In Sec. III, we show the numerical results for the differential distribution, and the implications to the measurement of the top quark mass and the searches for the anomalous Wtb couplings. Finally, in Sec. IV, we present our conclusion. 


\section{NLO CALCULATION}

In this section, we will describe the major theoretical details and numerical results for the total cross sections. The generic processes under consideration are $e^{-} \bar{b} \rightarrow \nu_{e} \bar{t} \rightarrow$ $\nu_{e} \ell^{-} \bar{\nu}_{\ell^{-}} \bar{b}$ and $e^{-} b \rightarrow \nu_{e} \ell^{-} \bar{\nu}_{\ell^{-}} b$. The leading-order (LO) Feynman diagrams are depicted in Fig. 1. Our computation is based on combination of GoSam2 . $0[100,101]$ and a native Monte Carlo framework. We use the GoSam2 . 0 program to generate one-loop results for the virtual amplitudes. We adopt the dipole subtraction method $[102,103]$ to construct local subtraction terms and their integrations for real emissions. All these ingredients are embedded in a MC program which can accomplish the cancellation of IR singularities and phase-space integrations.

In the following we introduce the dipole subtraction method used to deal with NLO calculations of subprocesses in single top quark production. We present the numerical result for the total cross section at the NLO based on the framework. The discussions on scale variations at both $\mathrm{LO}$ and NLO are shown at the end of this section.

\section{A. Theoretical framework}

We use the 5FS which treats the bottom quark as a massless parton in initial hadrons. The 5FS ensures a resummation of large quasicollinear logarithms from gluon splitting to bottom quarks through the parton distribution function of bottom quarks. The NLO cross section can be written as $[102,103]$
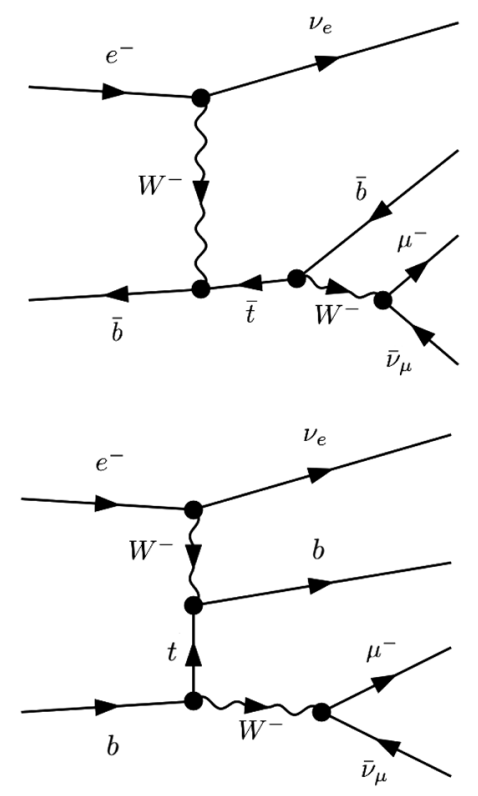

FIG. 1. LO Feynman diagrams for the single top quark production with leptonic decays at the $\mathrm{LHeC}$. We also include the subprocess that has an indistinguishable final state as the single top quark production.

$$
\begin{aligned}
& \sigma^{\mathrm{NLO}\{5\}}(p)+\sigma^{\mathrm{NLO}\{4\}}(p)+\int_{0}^{1} d x \hat{\sigma}^{\mathrm{NLO}\{4\}}(x ; x p) \\
& \equiv \int_{5}\left[\left(\mathrm{~d} \sigma^{\mathrm{R}}(p)\right)_{\epsilon=0}-\left(\sum_{\text {dipoles }} \mathrm{d} \sigma^{\mathrm{B}}(p) \otimes \mathrm{d} V_{\text {dipole }}\right)_{\epsilon=0}\right] \\
& \quad+\int_{4}\left[\mathrm{~d} \sigma^{\mathrm{V}}(p)+\mathrm{d} \sigma^{\mathrm{B}}(p) \otimes \boldsymbol{I}\right]_{\epsilon=0} \\
& \quad+\int_{0}^{1} d x \int_{4}\left[\mathrm{~d} \sigma^{\mathrm{B}}(x p) \otimes(\boldsymbol{P}+\boldsymbol{K})(x)\right]_{\epsilon=0}
\end{aligned}
$$

where the contributions $\sigma^{\mathrm{NLO}\{5\}}(p)$ and $\sigma^{\mathrm{NLO}\{4\}}(p)$ (with a five-body final state and four-body final state, respectively) represent the subtracted real contributions and virtual contributions including integrated dipoles. The third term $\int_{0}^{1} d x \hat{\sigma}^{\mathrm{NLO}\{4\}}(x ; x p)$ is a finite remainder which comes from the cancellation of the $\epsilon$ poles of the collinear counterterms. It contains an additional one-dimensional integration with respect to the longitudinal momentum fraction $x . \boldsymbol{P}$ and $\boldsymbol{K}$ are universal functions of $x$ which are finite for $\epsilon \rightarrow 0 . \mathrm{d} \sigma^{\mathrm{R}}, \mathrm{d} \sigma^{\mathrm{V}}$, and $\mathrm{d} \sigma^{\mathrm{B}}$ are the fully differential cross sections from real, virtual (one-loop), and Born contributions, respectively. The dipole factors $\mathrm{d} V_{\text {dipole }}$ describe the two-parton decays of the emitters. The factor $\boldsymbol{I}$ is derived from the dipole factors by integrating out a single parton phase space, which will cancel the $\epsilon$ poles in virtual contributions.

We show the one-loop Feynman diagrams with a $\bar{b}$ quark in the initial state in Fig. 2. There are similar one-loop diagrams for the process with a $b$ quark in the initial state which are omitted for simplicity. The real emission Feynman diagrams with a $\bar{b}$ quark or gluon in the initial state are shown in Figs. 3 and 4, respectively. The corresponding subtraction terms include those of initialstate emitter and final-state spectator $\mathcal{D}_{j}^{a i}$ and/or final-state emitter and initial-state spectator $\mathcal{D}_{i j}^{a}$,
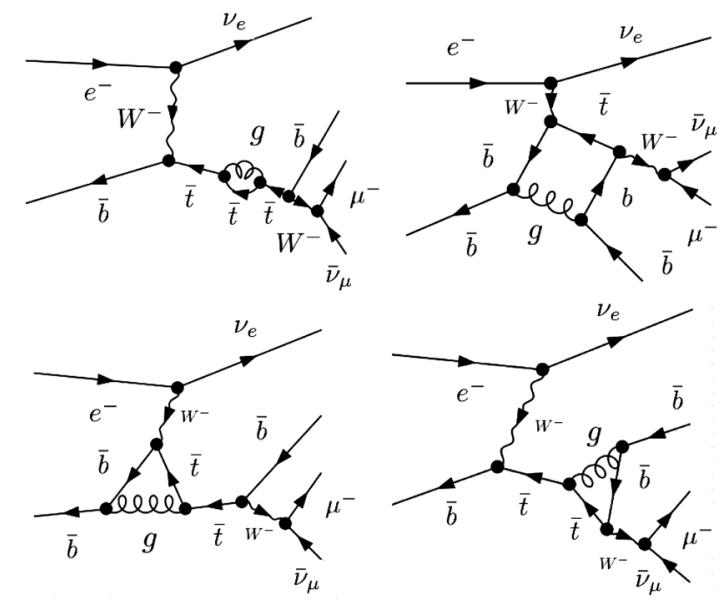

FIG. 2. One-loop Feynman diagrams for the single top quark production with leptonic decays at the $\mathrm{LHeC}$ with a $\bar{b}$ quark in the initial state. 


$$
\begin{gathered}
\mathcal{D}_{j}^{a i}\left(p_{1}, \ldots, p_{5} ; p_{a}, \ldots\right)=-\frac{1}{2 p_{a} p_{i}} \frac{1}{x_{i j, a}, a i}\left(\ldots, \tilde{j}, \ldots ; \tilde{a} i, \ldots\left|\frac{\boldsymbol{T}_{j} \cdot \boldsymbol{T}_{a i}}{\boldsymbol{T}_{a i}^{2}} \mathbf{V}_{j}^{a i}\right| \ldots, \tilde{j}, \ldots ; \tilde{a} i, \ldots\right\rangle_{4, \tilde{a}}, \\
\mathcal{D}_{i j}^{a}\left(p_{1}, \ldots, p_{5} ; p_{a}, \ldots\right)=-\frac{1}{2 p_{i} p_{j}} \frac{1}{x_{i j, a}}\left\langle\ldots, \tilde{i j}, \ldots ; \tilde{a}, \ldots\left|\frac{\boldsymbol{T}_{a} \cdot \boldsymbol{T}_{i j}}{\boldsymbol{T}_{i j}^{2}} \mathbf{V}_{i j}^{a}\right| \ldots, \tilde{i j}, \ldots ; \tilde{a}, \ldots\right\rangle_{4, a}, \\
x_{i j, a}=\frac{p_{a} p_{i}+p_{a} p_{j}-p_{i} p_{j}}{p_{a} p_{i}+p_{a} p_{j}} .
\end{gathered}
$$

The $\mathcal{D}_{j}^{a i}$ terms cancel the singularities of the matrix elements when the final-state parton $i$ and the initial-state parton become collinear. Similarly, the $\mathcal{D}_{i j}^{a}$ cancel the singularity of the matrix elements when the final-state gluon and $b$ or $\bar{b}$ become collinear. $\boldsymbol{T}$ and $\boldsymbol{V}$ are the color charge operator and spin function, respectively. The $|\ldots\rangle_{4, \tilde{a i}}$ and $|\ldots\rangle_{4, a}$ are reduced born matrix elements by replacing the parton pair $a, i$ with a single parton $\tilde{a} i$ and by replacing the parton pair $i, j$ with a single parton $\tilde{i j}$, respectively.

The calculations are carried out in the complex-mass scheme $[104,105]$ such that our results are valid in all regions of the phase space including when the top quark is off shell. The complex-mass scheme is a generalization of the on-shell renormalization scheme, in which case the bare top quark mass includes a complex renormalized mass $\mu_{t}^{2}=m_{t}^{2}-i m_{t} \Gamma_{t}$ and a complex counterterm $\delta \mu_{t}$. The cross sections are dominated by contributions from diagrams with a top quark resonance. However, we have also included the nonresonant diagrams, as can be seen in Figs. 1-4.
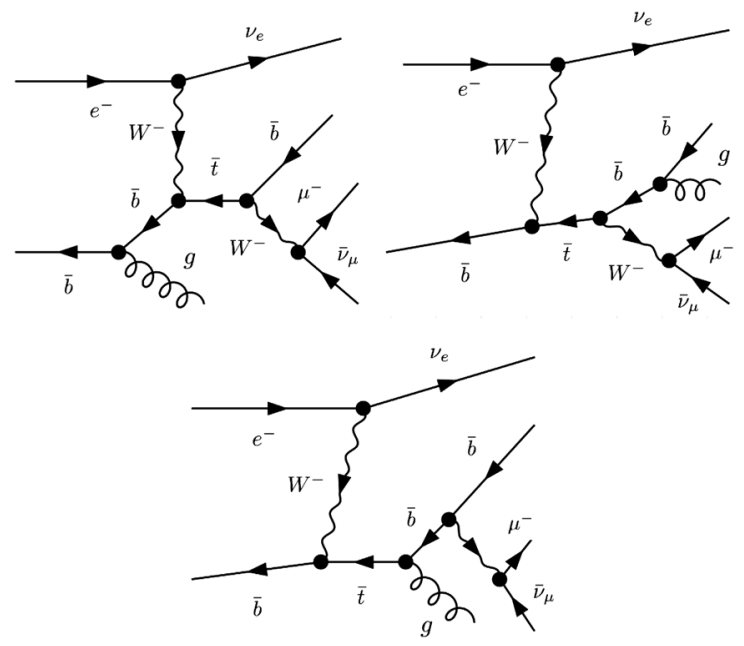

FIG. 3. Real emission Feynman diagrams for the single top quark production with leptonic decays at the $\mathrm{LHeC}$ with a $\bar{b}$ quark in the initial state.
We are now ready to make predictions for any infrared or collinear safe observable provided with the virtual corrections from GoSam2 . 0 and the formulas of the dipole terms in $[100,102]$. As for the parametrization of the phase space, we use the multichannel approach to accommodate for the singular structures of the top quark resonance. The numerical integrations are performed with the Monte Carlo library CUBA [106].

\section{B. Numerical result}

We study the single top quark production in $e p$ collisions at the $\mathrm{LHeC}$ with an electron beam energy of $70 \mathrm{GeV}$ and a proton beam energy of $7 \mathrm{TeV}$. We use the following set of SM parameters in the numerical calculations [44]:

$$
\begin{aligned}
m_{Z} & =91.1876 \mathrm{GeV}, \quad m_{t}=172.5 \mathrm{GeV}, \\
m_{W} & =80.385 \mathrm{GeV}, \quad G_{\mathrm{F}}=1.16639 \times 10^{-5} \mathrm{GeV}^{-2} .
\end{aligned}
$$

The CT18 next-to-next-to-leading-order (NNLO) parton distribution functions (PDFs) [107] and the strong coupling constant $\alpha_{s}\left(m_{Z}\right)=0.118$ are used throughout the calculations. The nominal choice of the factorization and

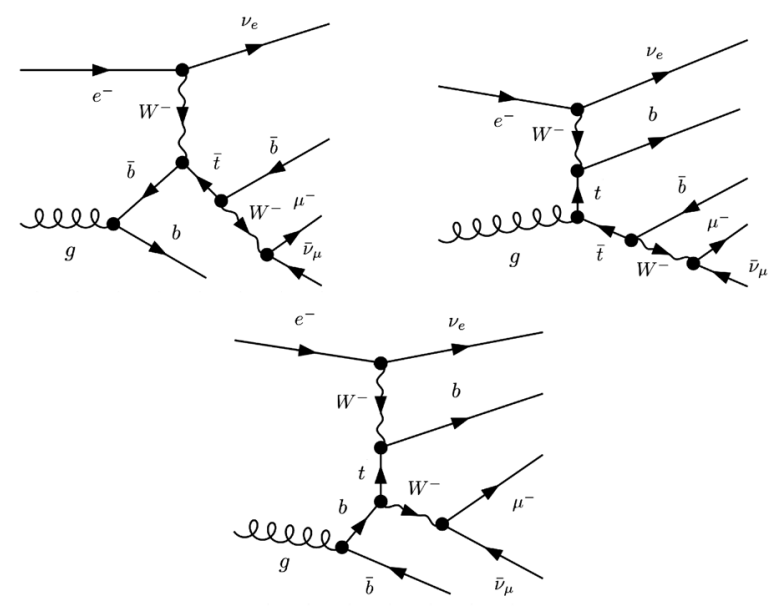

FIG. 4. Real emission Feynman diagrams for the single top quark production with leptonic decays at the $\mathrm{LHeC}$ with a gluon in the initial state. 
TABLE I. Inclusive cross sections for the single top quark production with leptonic decays at the $\mathrm{LHeC}$ at various orders in QCD with a nominal scale choice of $m_{t} / 2$. The scale variations are calculated by varying the scales from $\mu_{F}=\mu_{R}=m_{t} / 4$ to $m_{t}$ and are shown in percentages. In the numbers of cross sections the upper (lower) variation corresponds to the scale choice of $m_{t}$ $\left(m_{t} / 4\right)$. Separate contributions from three subprocesses with different initial states are also shown.

\begin{tabular}{lcc}
\hline \hline Inclusive $(\mathrm{pb})$ & $\mathrm{LO}$ & $\mathrm{NLO}$ \\
\hline$\sigma$ (total) & $0.281_{-11 \%}^{+8.2 \%}$ & $0.257_{+2.6 \%}^{+0.92 \%}$ \\
$\sigma[\bar{b}]$ & 0.281 & 0.264 \\
$\sigma[b]$ & $5.35 \times 10^{-4}$ & $5.18 \times 10^{-4}$ \\
$\sigma[g]$ & & $-6.97 \times 10^{-3}$ \\
\hline \hline
\end{tabular}

renormalization scales are $\mu_{R}=\mu_{F}=m_{t} / 2$, and the scale variations are calculated by varying the two scales simultaneously from $m_{t} / 4$ to $m_{t}$.

We list our predictions of the inclusive cross sections for the single top quark production with leptonic decays at different perturbative orders in Table I, with scale variations shown as percentages. We find that the LO cross section is dominated by the subprocess with a bottom antiquark in the initial state. The NLO QCD corrections reduce the total cross sections by $8.5 \%$. The full NLO corrections consist of three pieces from subprocesses with the bottom antiquark, bottom quark, and gluon in the initial state. The NLO corrections are also dominated by the subprocess with a bottom antiquark in the initial state. The scale variations at NLO are decreased by 5 times compared to LO and are within $3 \%$.

For the single top quark production, we apply various selection cuts to account for the finite kinematic coverage of the detectors and to suppress the SM background from the associated production of vector bosons with jets. Finalstate quarks and gluons are clustered into jets using the anti$k_{T}$ jet algorithm [108]. The jet-resolution parameter is set to 0.4 . We require at least one $b$-tagged jet in the final state. The cuts on the transverse momentum and pseudorapidity of the jet and lepton are shown below:

$$
\begin{aligned}
\left|\eta_{\ell^{-}}\right|<5, & p_{T, \ell^{-}}>10 \mathrm{GeV}, \\
\left|\eta_{j}\right|<5, & p_{T, \text { jet }}>30 \mathrm{GeV} .
\end{aligned}
$$

TABLE II. Similar to Table I but for fiducial cross sections.

\begin{tabular}{lcc}
\hline \hline Fiducial $(\mathrm{pb})$ & $\mathrm{LO}$ & $\mathrm{NLO}$ \\
\hline$\sigma$ (total) & $0.242_{-11 \%}^{+8.1 \%}$ & $0.205_{+3.1 \%}^{+0.76 \%}$ \\
$\sigma[\bar{b}]$ & $0.242^{-4}$ & 0.207 \\
$\sigma[b]$ & $5.01 \times 10^{-4}$ & $4.62 \times 10^{-4}$ \\
$\sigma[g]$ & & $-2.95 \times 10^{-3}$ \\
\hline \hline
\end{tabular}

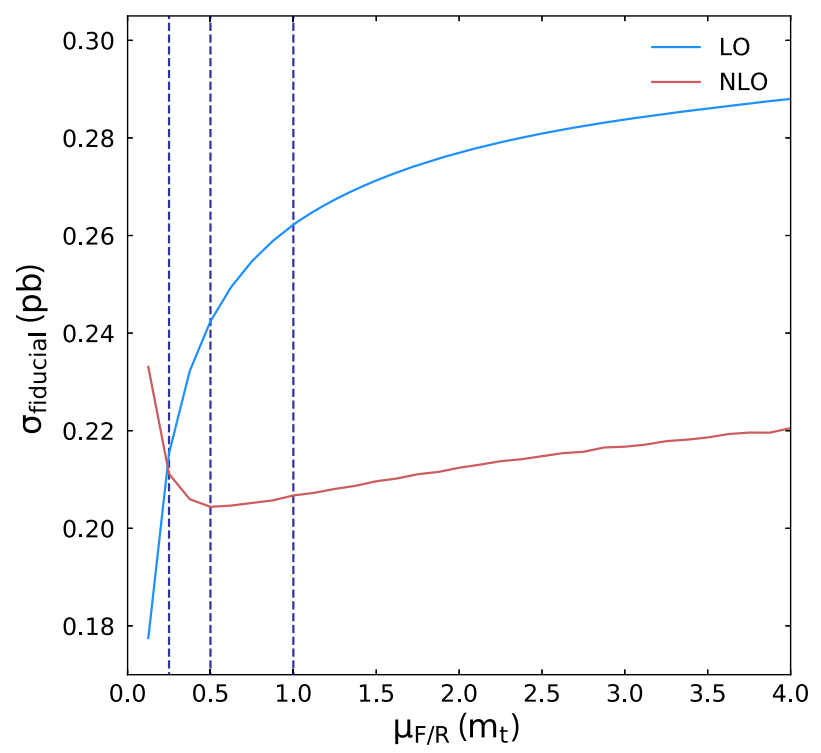

FIG. 5. The dependence of the fiducial cross section on the factorization and renormalization scales $\left(\mu_{F}=\mu_{R}\right)$.

We show the predictions for the fiducial cross sections at LO and NLO in Table II. We list contributions from the different subprocesses and show the scale variations in percentages. We find that the selection cuts reduce the cross section by about $14 \%$ at LO in comparison to Table I. The QCD corrections are still dominated by contributions from the subprocess with a $\bar{b}$ quark in the initial state. The full NLO corrections reduce the fiducial cross section by $15 \%$. The scale variations of the NLO predictions are about $3 \%$, largely reduced compared to the $\mathrm{LO}$ ones. We can also calculate the efficiency, which is defined as the ratio of the fiducial cross section to the inclusive cross section. From the results in Tables I and II, we derive the efficiencies as 0.861 and 0.798 at LO and NLO, respectively.

The dependence of the fiducial cross section on the factorization and renormalization scales $\left(\mu_{F}=\mu_{R}\right)$ are shown in Fig. 5. The three vertical lines correspond to scales $m_{t} / 4, m_{t} / 2$, and $m_{t}$, respectively. The two curves denote cross sections at LO and NLO. The cross sections change dramatically below the scale $m_{t} / 4$. We find that the cross sections are more stable against the scale choice in the range $m_{t} / 4<\mu_{F / R}<m_{t}$. This motivates our nominal choice of the scale and its variation range.

TABLE III. Similar to Table I but for fiducial cross sections with more stringent cuts.

\begin{tabular}{lcc}
\hline \hline Fiducial $(\mathrm{pb})$ & LO & NLO \\
\hline$\sigma$ (total) & $0.132_{-11 \%}^{+8.2 \%}$ & $0.108_{+4.2 \%}^{+0.14 \%}$ \\
$\sigma[\bar{b}]$ & 0.132 & 0.104 \\
$\sigma[b]$ & $4.08 \times 10^{-4}$ & $3.63 \times 10^{-4}$ \\
$\sigma[g]$ & & $3.71 \times 10^{-3}$ \\
\hline \hline
\end{tabular}


Table III shows the predictions for the fiducial cross sections at the LO and NLO, with more stringent cuts shown below:

$$
\begin{aligned}
\left|\eta_{\ell^{-}}\right|<3.5, & p_{T, \ell^{-}}>25 \mathrm{GeV} \\
\left|\eta_{j}\right|<3.5, & p_{T, \text { jet }}>50 \mathrm{GeV} .
\end{aligned}
$$

The cuts reduce the cross section by about $53 \%$ at $\mathrm{LO}$ in comparison to Table I. The scale variations of the LO prediction are identical to those shown in Table I. The NLO corrections reduce the fiducial cross section by about $18 \%$ with scale variations slightly larger than those in Table II. We use the selection cuts in Eq. (6) as our default setup in the rest of the paper.

\section{PHENOMENOLOGICAL ANALYSIS}

In this section, we present a phenomenological analysis of the differential distributions based on our MC program. We show the QCD corrections and theoretical uncertainties of the distributions. We propose a method for determining the top quark mass using the average transverse momentum of the charged lepton and estimate various uncertainties of the measurement. We also show the impact of the QCD corrections on constraining the anomalous couplings of the top quark.

\section{A. LO and NLO predictions}

We present predictions for various distributions within the fiducial region defined in Sec. II B in Figs. 6-10. In the plot of each distribution, the upper panel shows the LO and NLO distributions with a nominal scale choice of $m_{t} / 2$ and alternative scale choices of $m_{t} / 4$ and $m_{t}$. The middle panel shows the ratio of the NLO predictions to LO predictions $\left(d \sigma_{\mathrm{NLO}} / d \sigma_{\mathrm{LO}}\right)$ with each scale choice. The lower panel shows the PDF uncertainties and scale variations at both LO and NLO. The PDF uncertainties at $68 \%$ C.L. are calculated at LO using the 58 error PDF sets in the CT18 NNLO PDFs [107] and are normalized to the LO distributions with the scale choice $m_{t} / 2$. The scale variations of the LO (NLO) predictions are also shown in the lower panel and are normalized to the LO (NLO) predictions with the scale choice $m_{t} / 2$.

The distribution of the transverse momentum of the charged lepton is presented in Fig. 6. In the upper panel, the peak of the distribution occurs at around $25 \mathrm{GeV}$, and the $P_{T}$ of the charged lepton can extend to $100 \mathrm{GeV}$. The NLO corrections decrease the normalization of the distribution without changing the position of the peak. The NLO distributions of three different scales are closer to each other than the LO ones because of the weaker scale dependence of the NLO predictions. In the middle panel we can see a better perturbative convergence for the scale of $m_{t} / 4$ in the low $P_{T}$ region, where the ratio $d \sigma_{\mathrm{NLO}} / d \sigma_{\mathrm{LO}}$ is close to 1 . In the lower panel, the scale variations at LO are
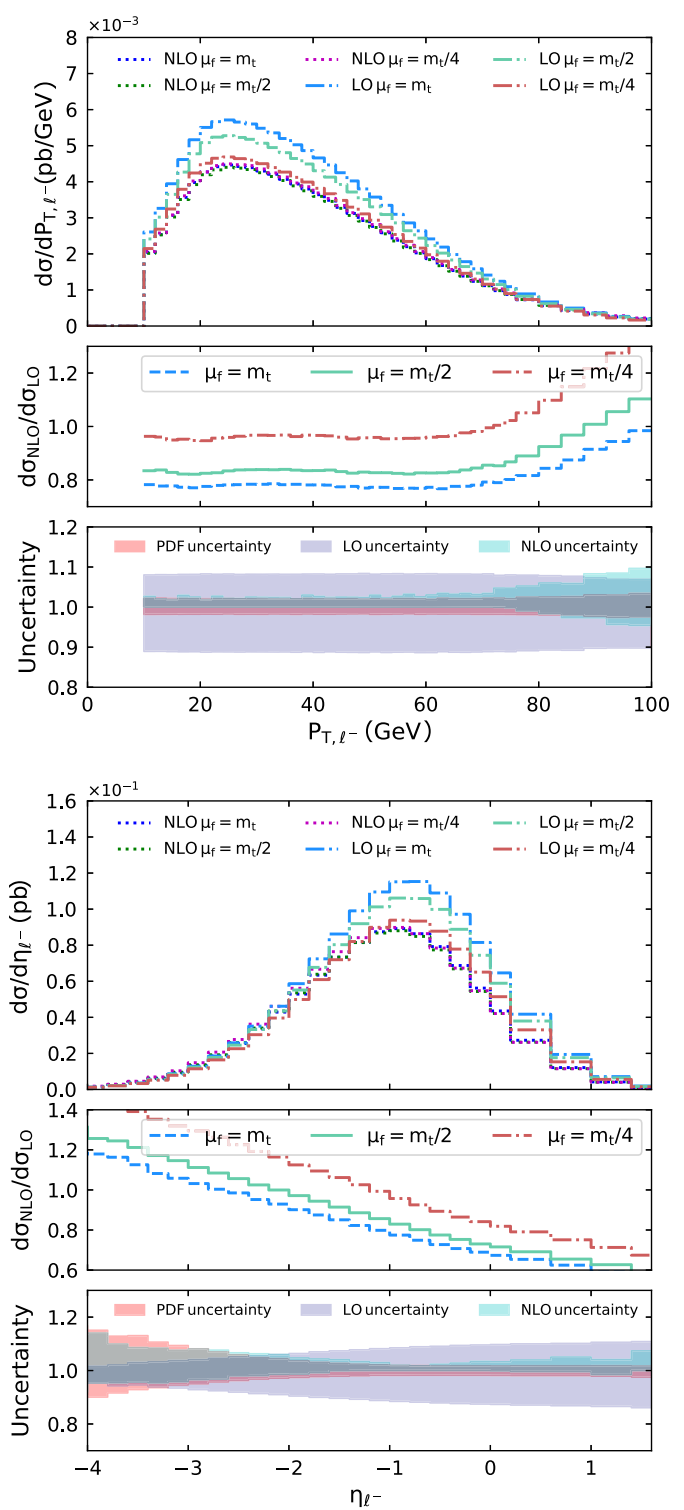

FIG. 6. Transverse momentum and pseudorapidity distributions of the charged lepton with fiducial cuts applied at LO and NLO with a nominal scale choice of $m_{t} / 2$ and alternative scale choices of $m_{t} / 4$ and $m_{t}$. The middle panel shows the ratio of the NLO predictions to LO predictions $\left(d \sigma_{\mathrm{NLO}} / d \sigma_{\mathrm{LO}}\right)$ with each scale choice. The lower plot shows the PDF uncertainties and scale variations at both LO and NLO.

much larger than the PDF uncertainties. The NLO corrections reduce the scale variations significantly to a level that is comparable to or even smaller than the PDF uncertainties. Figure 6 also shows the pseudorapidity distribution of the charged lepton. The peak of the distribution occurs at a negative value due to the asymmetric collision. For all three scale choices the ratio of NLO to LO distribution decreases with the pseudorapidity. The scale variations at NLO are close to the PDF uncertainties in the full range of pseudorapidity. 

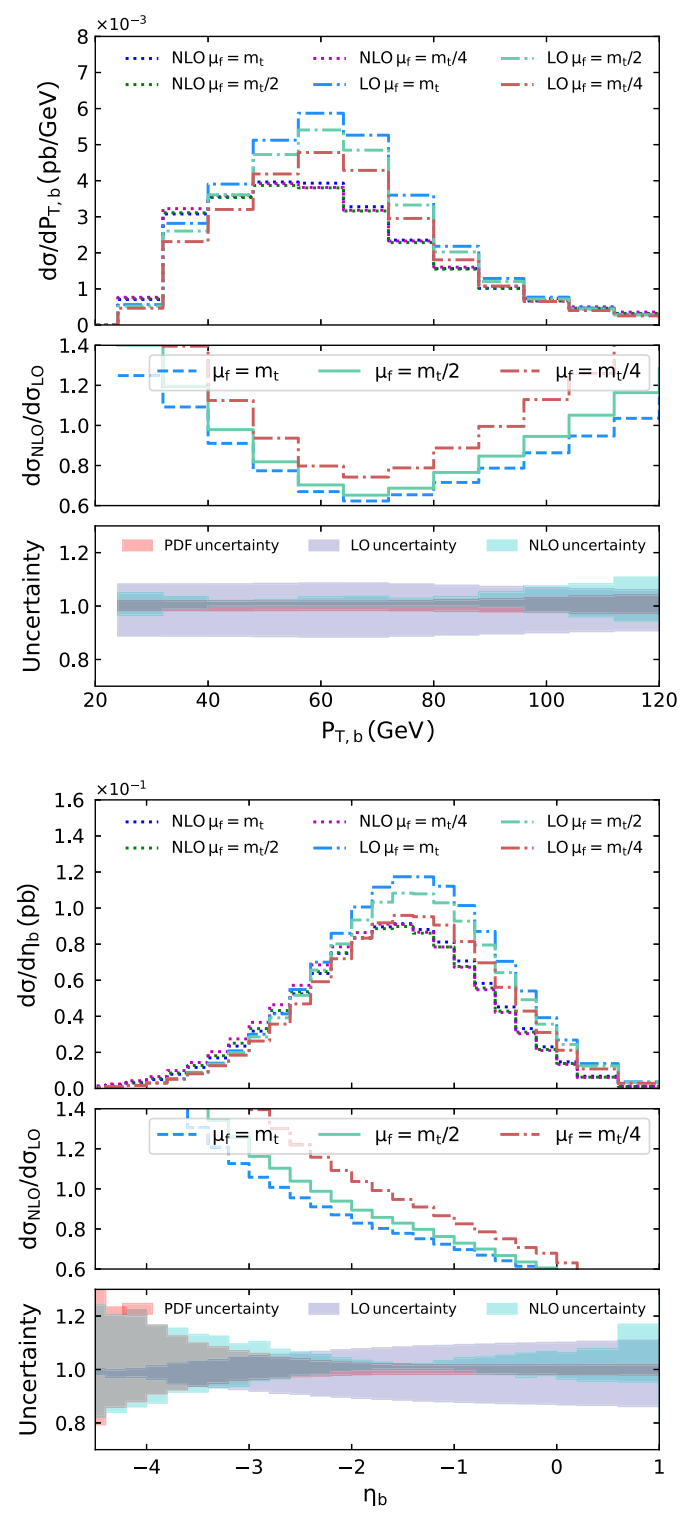

FIG. 7. Similar to Fig. 6 but for the transverse momentum and pseudorapidity distributions of the b-jet.

We show the transverse momentum and pseudorapidity distributions of the b-jet in Fig. 7. In the transverse momentum distribution, the peak of the distribution occurs at around $60 \mathrm{GeV}$. The NLO corrections decrease the normalization of the transverse momentum distribution and shift the peak position to lower $P_{T}$. The ratios of the NLO to LO distributions show a minimum at $P_{T}$ close to the peak region. These behaviors are due to the nonresonant contributions and the hard gluon radiations. Pseudorapidity distributions of the b-jet are similar to the distributions of the charged lepton. They peak at negative values of $\eta_{b}$. However, the ratios of the NLO to LO predictions in the middle panel decrease more dramatically than in the cases of the charged lepton. The scale variations are largely reduced at the NLO in the low $\left|\eta_{b}\right|$ region. For large $\left|\eta_{b}\right|$ the scale variations at the $\mathrm{LO}$ are severely underestimated.

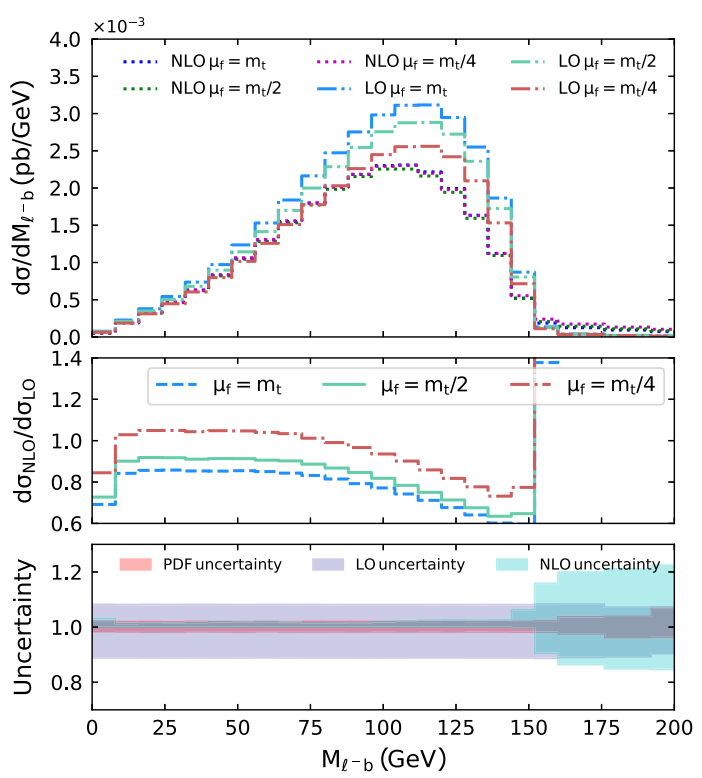

FIG. 8. Similar to Fig. 6 but for the invariant mass distributions of the charged lepton and b-jet system $\left(M_{\ell^{-} b}\right)$.

Figure 8 depicts distributions of the invariant mass from the system of the charged lepton and the b-jet (the visible decay products of the top quark). The sharp cutoff in the distribution is due to the kinematic constraint $M_{\ell^{-} b}^{2}<M_{t}^{2}-M_{W}^{2} \approx(150 \mathrm{GeV})^{2}$. The ratios of the NLO to LO distributions increase right after the cutoff because of the nonresonant and off-shell effects at NLO. This distribution has been used to measure the top quark mass in the single top quark production at the LHC $[109,110]$. We present the

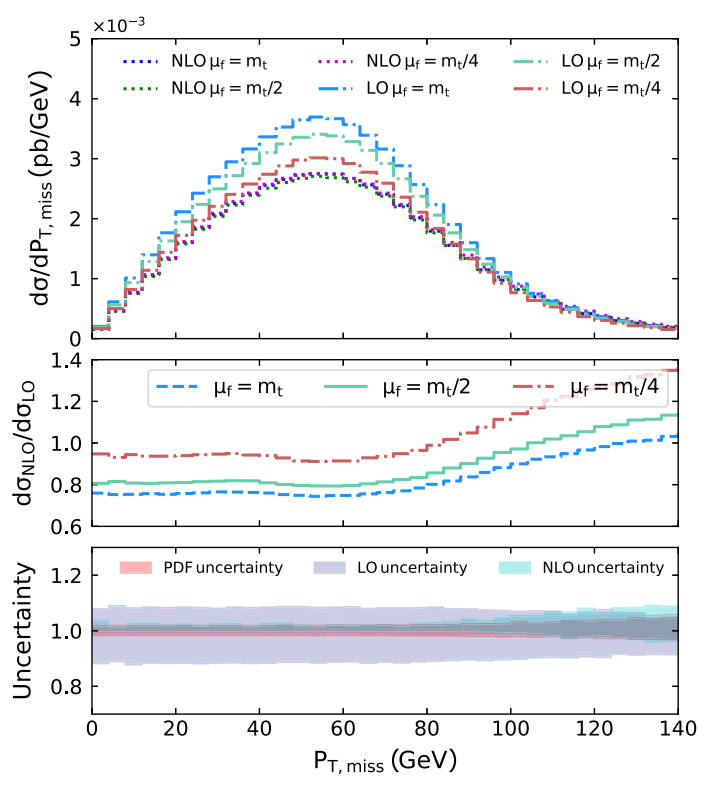

FIG. 9. Similar to Fig. 6 but for the distribution of the missing transverse momentum $\left(P_{T, \text { miss }}=\left|P_{T, \nu_{\ell^{-}}}+P_{T, \bar{\nu}_{e}}\right|\right)$ distribution. 

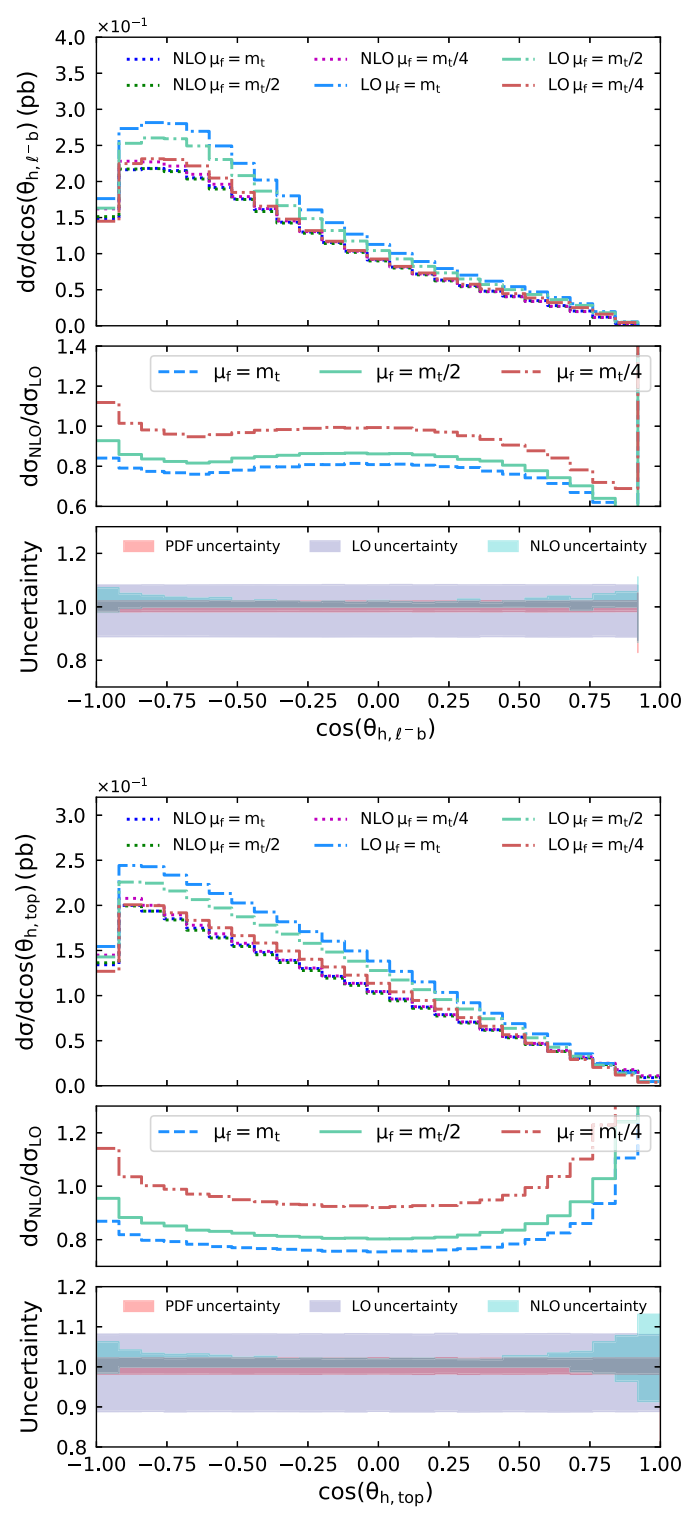

FIG. 10. Similar to Fig. 6 for the distributions of helicity angles of the visible-particles-reconstructed top quark and the truth top quark.

distribution of the total missing transverse momentum $\left(P_{T, \text { miss }}=\left|P_{T, \nu_{\ell^{-}}}+P_{T, \bar{\nu}_{e}}\right|\right)$ in Fig. 9. The peak of the distribution occurs at a much larger $P_{T}$ compared to the distribution of $P_{T, \ell^{-}}$. The ratio $d \sigma_{\mathrm{NLO}} / d \sigma_{\mathrm{LO}}$ rises significantly in the tail region. The PDF uncertainties and scale variations at $\mathrm{NLO}$ are much smaller than the scale variations at LO.

For the leptonic decays of the top quark, the charged lepton $\ell^{-}$is strongly correlated with the spin direction of the top quark. We define the helicity angle $\theta_{h}$ as the angle between the direction of the momentum of the charged lepton and the spin of the top quark in the rest frame of the top quark [111,112]. In the single top quark production, the spin of the top antiquark is always in the opposite direction

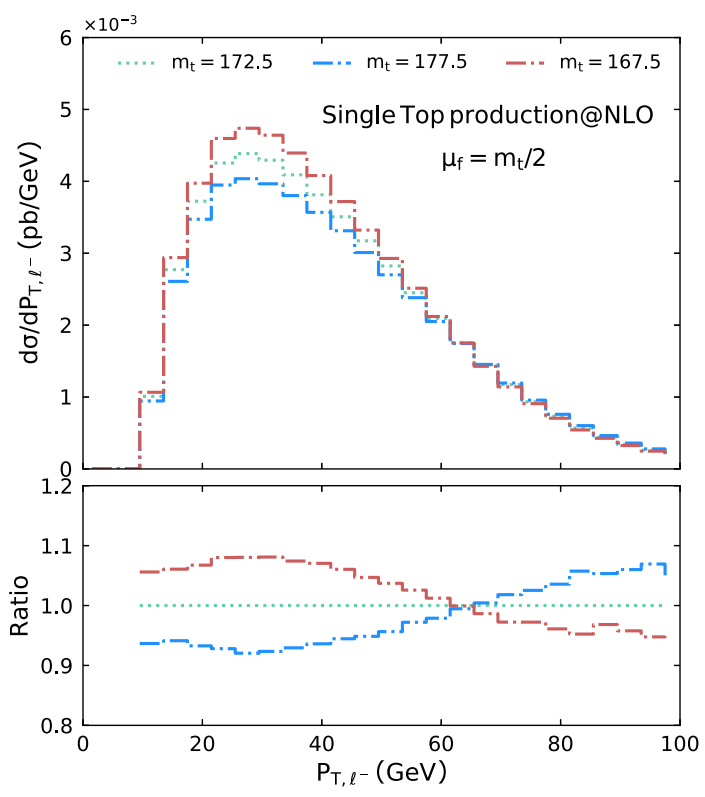

FIG. 11. Transverse momentum distribution of the charged lepton with the fiducial cuts applied for different choices of the top quark mass at NLO in QCD.

of the momentum of the incident electron. We show the distributions of the helicity angles in Fig. $10 . \cos \left(\theta_{h, \ell^{-} b}\right)$ is the cosine of the helicity angle based on the top quark reconstructed with only visible decay products and the $\cos \left(\theta_{h \text {,top }}\right)$ based on the truth top quark. The $\cos \left(\theta_{h, \text { top }}\right)$ distribution displays a strong correlation pattern except in the extreme backward region, where the fiducial cuts play an important role. The correlations are weakened in the distribution of $\cos \left(\theta_{h, \ell^{-} b}\right)$ due to the approximation with the reconstructed top quark. The ratios $d \sigma_{\mathrm{NLO}} / d \sigma_{\mathrm{LO}}$ are flat in most regions of the helicity angles.

\section{B. Top quark mass}

We study the extraction of the top quark mass using the transverse momentum distribution of the charged lepton, following our previous work [113]. We show the sensitivity of the distributions of $P_{T, \ell^{-}}$to the top quark mass in Fig. 11. The three curves correspond to results for the top quark masses of $167.5,172.5$, and $177.5 \mathrm{GeV}$, respectively, with the nominal scale choice. The larger top quark mass results in a harder $P_{T, \ell^{-}}$distribution and also a lower overall normalization. We choose the average $P_{T, \ell^{-}}$in the fiducial region as our principle observable to extract the top quark mass. We also study the sensitivity of the total fiducial cross section on the top quark mass.

The results for the average $P_{T}$ of the charged lepton, including their scale variations, are presented in Table IV. The LO results are less affected by scale choices because the shape of the distribution depends weakly on the scale at LO. It shows that the LO predictions are not reliable to 
TABLE IV. Average transverse momentum of the charged lepton at various orders in QCD with a central scale choice of $m_{t} / 2$. The superscript (subscript) corresponds to the variation with the scale $m_{t}\left(m_{t} / 4\right)$.

\begin{tabular}{lcc}
\hline \hline $\mathrm{GeV}$ & LO & NLO \\
\hline$\left\langle p_{T, \ell^{-}}\right\rangle$ & $39.41_{+0.006}^{-0.01}$ & $39.75_{+0.16}^{-0.11}$ \\
\hline \hline
\end{tabular}

extract the top quark mass, as can be seen in the gap between the LO and NLO predictions. The NLO scale variations nearly reach the level of changes induced by varying the top quark mass by $1 \mathrm{GeV}$, which is shown in Table V.

We further investigate the parametric uncertainties of the average $P_{T, \ell^{-}}$due to parton distribution functions, bottom quark mass and $\alpha_{S}$ in Table V. The PDF uncertainties are calculated using the CT18NNLO PDFs. In the 5FS, the dependence of the cross section on the bottom quark mass arises entirely from the bottom quark PDF which resums the large logarithms of $\alpha_{S} \ln \left(Q^{2} / m_{b}^{2}\right)$. We study the bottom quark mass dependence of our predictions using the MMHT2014NNLO PDF set [114] with a range of $m_{b}$ values since in CT18 there are no such sets with bottom quark mass variations. We use three MMHT PDF sets encapsulated in LHAPDF6 [115] with bottom quark masses of $4.25,4.75$, and $5.25 \mathrm{GeV}$. The resulting variations of the average $P_{T, \ell^{-}}$with respect to the one using a nominal MMHT PDF set are reported in Table V and are negligible. We expect the relative variations induced by the bottom quark mass not to be sensitive to the PDF families used, since they are mostly of perturbative origin. We calculate the dependence on the QCD coupling constant by varying $\alpha_{S}\left(m_{Z}\right)$ with $0.118 \pm 0.001$. We estimate the statistical error assuming that the $\mathrm{LHeC}$ can achieve a total integrated luminosity of $100 \mathrm{fb}^{-1}$. We conclude that the variations of the average $P_{T, \ell^{-}}$by varying the top quark mass with $1 \mathrm{GeV}$ are significantly higher than the other parametric uncertainties. The statistical error is of a similar size as the variation from the top quark mass. We show similar results for the total fiducial cross section in Table VI. In contrast to the case of average $P_{T, \ell^{-}}$, the parametric uncertainties are much larger than the variation

TABLE V. Various parametric uncertainties, the expected statistical error, and the dependence on the top quark mass of the average $P_{T, \ell^{-}}$. The parameters are varied by the values in parentheses.

\begin{tabular}{lc}
\hline \hline $\mathrm{GeV}$ & $\delta\left\langle p_{T, \ell^{-}}\right\rangle$ \\
\hline PDFs $(68 \%$ C.L. $)$ & $+0.0126-0.0081$ \\
$\alpha_{S}\left(m_{Z}\right)(0.001)$ & $+0.0034-0.0031$ \\
$m_{b}(0.5 \mathrm{GeV})$ & \pm 0.0011 \\
Statistical error & 0.1341 \\
$m_{t}(1.0 \mathrm{GeV})$ & 0.1225 \\
\hline \hline
\end{tabular}

TABLE VI. Similar to Table V but for the total fiducial cross section. The variations of the cross section are shown as percentages.

\begin{tabular}{lc}
\hline \hline$\%$ & $\delta \sigma$ \\
\hline PDFs $(68 \%$ C.L. $)$ & $+2.50-2.08$ \\
$\alpha_{S}\left(m_{Z}\right)(0.001)$ & $+1.87-1.43$ \\
$m_{b}(0.5 \mathrm{GeV})$ & \pm 4.86 \\
Statistical errors & 0.698 \\
$m_{t}(1.0 \mathrm{GeV})$ & 0.950 \\
\hline \hline
\end{tabular}

due to the top quark mass. It shows about a $1 \%$ change by varying the top quark mass with $1 \mathrm{GeV}$, while the PDF uncertainties are about $2 \%$. The variations due to the bottom quark mass are about $5 \%$ if one varies $m_{b}$ by $0.5 \mathrm{GeV}$, and $2 \%$ if one varies $m_{b}$ by $0.2 \mathrm{GeV}$, as recommended in Ref. [116].

We use a linear model on dependence of the average $P_{T, \ell^{-}}$on the top quark mass

$$
\left\langle p_{T, \ell^{-}}\right\rangle=p_{T, 0}+\lambda\left[\frac{m_{t}}{\mathrm{GeV}}-172.5\right]
$$

where $p_{T, 0}$ is the average transverse momentum of the charged lepton for a top quark mass of $172.5 \mathrm{GeV}$. The values of $\lambda$ and $p_{T, 0}$ can be obtained from the results in Tables IV and V. The top quark mass can be extracted from the measurement of $\left\langle p_{T, \ell^{-}}\right\rangle$. In the following, we estimate the uncertainties of the extracted top quark mass due to both the statistical error and the theoretical uncertainties. We neglect the contributions from various parametric uncertainties, which are small as mentioned earlier.

We present the projection of the top quark mass measurement in Fig. 12 with a hypothetical value of 172.5 GeV. The horizontal line corresponds to the $\left\langle p_{T, \ell^{-}}\right\rangle$for a top quark mass of $172.5 \mathrm{GeV}$. The horizontal band represents the estimated statistical error of $\left\langle p_{T, \ell^{-}}\right\rangle$. The diagonal blue line is the NLO prediction on $\left\langle p_{T, \ell^{-}}\right\rangle$as a function of the top quark mass. The red band surrounding the diagonal line represents the scale variations of $\left\langle p_{T, \ell^{-}}\right\rangle$at the NLO. The uncertainties on the extracted top quark mass are computed while assuming linear error propagation and are represented by various vertical lines. The vertical lines of the statistical error are obtained by intersections of the horizontal band and the diagonal line. The vertical lines of the theoretical uncertainty are obtained as intersections of the diagonal band and the horizontal line. Finally, the statistical error of the extracted top quark mass amounts to $1.1 \mathrm{GeV}$ and the theoretical uncertainty amounts to +1.3 and $-0.9 \mathrm{GeV}$. The current uncertainties of the direct measurements of the top quark mass at the LHC are about $500-600 \mathrm{MeV}[44,117]$. The errors of indirect determinations of $m_{t}$ are about 1 to $2 \mathrm{GeV}$ [44]. As for the 


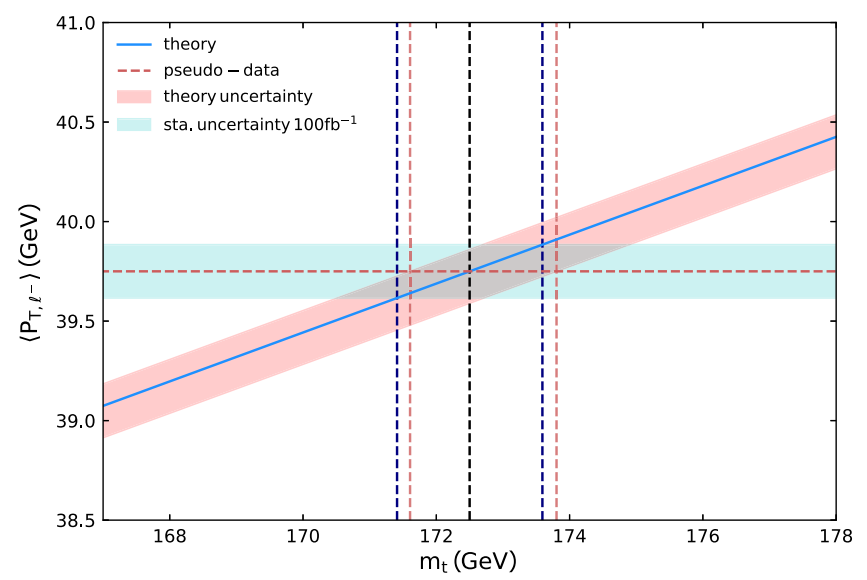

FIG. 12. Projection of the top quark mass measurement with a hypothetical value of $172.5 \mathrm{GeV}$. The diagonal line is the NLO prediction on $\left\langle p_{T, \ell^{-}}\right\rangle$as a function of the top quark mass. The diagonal band represents the scale variations of $\left\langle p_{T, \ell^{-}}\right\rangle$at NLO. The horizontal band represents the estimated statistical error of $\left\langle p_{T, \ell^{-}}\right\rangle$. Vertical lines indicate various uncertainties of the extracted top quark mass.

HL-LHC, the uncertainties of the direct measurements can be reduced to about $200 \mathrm{MeV}$ [117]. These measurements are from top quark pair production. Our proposed determination of $m_{t}$ is based on the single top quark production with only leptonic observables and has a precision similar to the LHC indirect measurements.

\section{Signal of new physics}

We study the possible improvement on searches of new physics with the NLO predictions of the single top quark production with leptonic decays. It has been shown that the $\mathrm{LHeC}$ can provide a better assessment of the effective $W t b$ couplings through the measurement of the single top quark production [33]. We can write the effective $W t b$ vertex, including SM contributions and those from new physics, as [118]

$$
\begin{aligned}
\mathcal{L}_{t b W}= & -\frac{g}{\sqrt{2}} \bar{b}\left(\gamma^{\mu}\left(\left(V_{t b}+\delta V_{L}\right) P_{L}+V_{R} P_{R}\right)\right. \\
& \left.-\frac{i \sigma^{\mu \nu} q_{\nu}}{m_{W}}\left(g_{L} P_{L}+g_{R} P_{R}\right)\right) t W_{\mu}^{-}+\text {H.c. }
\end{aligned}
$$

The new physics contributions can be due to dimension- 6 effective operators [119,120], for example [121],

$$
\begin{aligned}
& \frac{1}{\Lambda^{2}}\left\{C_{\phi q} O_{\phi q}^{(3,33)}+\left[C_{\phi \phi} O_{\phi \phi}^{33}+C_{t w} O_{u W}^{33}\right.\right. \\
& \left.\left.\quad+C_{b W} O_{d W}^{33}+\text { H.c. }\right]\right\},
\end{aligned}
$$

where $\phi$ is the SM Higgs doublet. The definition of the operators can be found in [121]. In this case the effective
TABLE VII. SM predictions on asymmetries of various observables at both LO and NLO with a nominal scale choice of $m_{t} / 2$. The numbers correspond to asymmetries $A(\Delta \eta, 0)$, $A(\Delta \varphi, \pi / 2)$, and $A(\cos , 0)$, respectively. The upper (lower) variation corresponds to an alternative scale of $m_{t}\left(m_{t} / 4\right)$. The statistical errors are calculated by assuming a total integrated luminosity of $100 \mathrm{fb}^{-1}$.

\begin{tabular}{lccc}
\hline \hline Observable & LO & NLO & Statistical error \\
\hline$\Delta \eta\left(b, \ell^{-}\right)$ & $-0.374_{+0}^{-0}$ & $-0.411_{-0.008}^{+0.007}$ & 0.006 \\
$\Delta \varphi\left(b, \ell^{-}\right)$ & $0.420_{-0.002}^{+0.001}$ & $0.388_{-0.002}^{+0.004}$ & 0.006 \\
$\Delta \varphi\left(b, E_{T}\right)$ & $0.805_{+0}^{-0}$ & $0.746_{-0.013}^{+0.012}$ & 0.005 \\
$\Delta \varphi\left(\ell^{-}, E_{T}\right)$ & $0.346_{-0}^{+0}$ & $0.292_{+0.008}^{-0.0010}$ & 0.007 \\
$\cos \left(b, \ell^{-}\right)$ & $0.419_{+0.011}^{-0.009}$ & $0.548_{+0.018}^{-0.018}$ & 0.006 \\
\hline \hline
\end{tabular}

couplings can be related to the corresponding operator coefficients as

$$
\begin{aligned}
\delta V_{L} & =\frac{1}{2} \frac{v^{2}}{\Lambda^{2}} C_{\phi q}, & g_{R} & =\sqrt{2} \frac{v^{2}}{\Lambda^{2}} C_{t W}, \\
V_{R} & =\frac{1}{2} \frac{v^{2}}{\Lambda^{2}} C_{\phi \phi}, & g_{L} & =\sqrt{2} \frac{v^{2}}{\Lambda^{2}} C_{b W} .
\end{aligned}
$$

It has been shown that the asymmetry of various observables can be sensitive to the modified Wtb couplings. It is defined as [33]

$$
A\left(X, X_{0}\right)=\frac{\sigma\left(X>X_{0}\right)-\sigma\left(X<X_{0}\right)}{\sigma\left(X>X_{0}\right)+\sigma\left(X<X_{0}\right)},
$$

where $\mathrm{X}$ is the kinematic observable and $X_{0}$ is the reference value. We consider the asymmetry of various observables including $\Delta \eta\left(b, \ell^{-}\right), \Delta \varphi\left(b, \ell^{-}\right), \Delta \varphi\left(b, E_{T}^{\prime}\right), \Delta \varphi\left(\ell^{-}, E_{T}\right)$, and $\cos \left(b, \ell^{-}\right)$, which are the separation of the pseudorapidities and azimuth angles, and the cosine angle of the reconstructed objects within the fiducial region. We present the SM predictions on the asymmetries $A(\Delta \eta, 0)$, $A(\Delta \varphi, \pi / 2)$, and $A(\cos , 0)$ at both LO and NLO with scale variations in Table VII. The upper (lower) variation corresponds to an alternative scale of $m_{t}\left(m_{t} / 4\right)$. The statistical errors are calculated by assuming a total integrated luminosity of $100 \mathrm{fb}^{-1}$. It shows that the NLO corrections are about $7 \%-30 \%$, depending on the observables. The scale variations at the LO largely underestimate the perturbative uncertainties. The scale variations at NLO are about $1 \%-3 \%$ at the same level as the statistical errors.

We can set constraints on the new physics by comparing the SM predictions with the projected measurements. We calculate the contributions from new physics to the single top quark production with leptonic decays at LO using MG5_aMC@NLO [122]. The generated events are analyzed with MadAnalysis5 [123]. We study the effects of the effective $W t b$ couplings $\delta V_{L}$ and $g_{R}$ using the model 
TABLE VIII. Projected bounds on the effective couplings $g_{R}$ and $\delta V_{L}$ at the $95 \%$ C.L. using different theory predictions by varying the couplings one at a time. The bounds are derived from the measurements on either the total fiducial cross section or the asymmetry $A\left(\Delta \eta\left(b, \ell^{-}\right), 0\right)$. The statistical errors are included while assuming a total integrated luminosity of $100 \mathrm{fb}^{-1}$. We further include an experimental uncertainty of $3 \%$ for the measurements of both the fiducial cross section and the asymmetry.

\begin{tabular}{lcccc}
\hline \hline & LO & LO + Scale variation & NLO & NLO + Scale variation \\
\hline$g_{R}\left(\sigma_{\text {tot }}\right)$ & {$[-0.047,0.041]$} & {$[-0.236,0.138]$} & {$[-0.039,0.036]$} & {$[-0.057,0.050]$} \\
$g_{R}\left(\Delta \eta\left(b, \ell^{-}\right)\right)$ & {$[-0.083,0.062]$} & {$[-0.083,0.062]$} & {$[-0.060,0.051]$} & {$[-0.071,0.059]$} \\
$\delta V_{L}\left(\sigma_{\text {tot }}\right)$ & {$[-0.0086,0.0083]$} & {$[-0.034,0.030]$} & {$[-0.0073,0.0071]$} & {$[-0.010,0.010]$} \\
\hline \hline
\end{tabular}

SMEFT@NLO [124] while assuming the effective couplings to be real numbers. The input parameters and fiducial cuts are the same as those in Sec. II B.

We show the projected bounds on the effective couplings $g_{R}$ and $\delta V_{L}$ at the $95 \%$ C.L. using different theory predictions by varying the couplings one at a time in Table VIII. The bounds are derived from the measurements on either the total fiducial cross section or the asymmetry $A\left(\Delta \eta\left(b, \ell^{-}\right), 0\right)$. The statistical errors assume a total integrated luminosity of $100 \mathrm{fb}^{-1}$. We further include an experimental uncertainty of $3 \%$ for the measurements of both the fiducial cross section and the asymmetry. The theoretical predictions are based on the new physics contributions calculated at LO combined with the SM predictions at LO (NLO) and while including (not including) the scale variations.

We find that the NLO corrections improve the constraints on the new physics in general due to both the shift of the SM central predictions and the reduction of the scale variations. At LO, when including the scale variations, the bounds on $\sigma_{\text {tot }}$ are enlarged by a factor of almost 4 . The NLO predictions result in more reliable bounds. The asymmetry $A\left(\Delta \eta\left(b, \ell^{-}\right), 0\right)$ yields slightly weaker bounds on $g_{R}$ in general than the total fiducial cross section does. For the bounds on $g_{R}$ derived from the cross section, the effects of scale variations are larger than in the case of the asymmetry. We have also compared our results with those in [33]. Our bounds on $\delta V_{L}$ and $g_{R}$ based on the LO predictions without the scale variations are almost identical to theirs. Note that the fiducial cuts used in our study and theirs are slightly different. To summarize we anticipate our best bounds on $g_{R}$ and $\delta V_{L}$ at the $95 \%$ C.L. being $[-0.057,0.050]$ and $[-0.010,0.010]$, respectively, as derived using the NLO SM predictions with scale variations. The sensitivity of $g_{R}$ is $[-0.05,0.02]$ at the $95 \%$ C.L. at the HL-LHC [117]. We conclude that the constraint on the coupling $g_{R}$ at the LHeC is weaker than the HL-LHC projection. The sensitivity of $\delta V_{L}$ is $[-0.036,0.036]$ at the $68 \%$ C.L. at the HL-LHC, as in Ref. [33]. We expect the LHeC to give a better constraint on $\delta V_{L}$ than the HL-LHC.

\section{CONCLUSIONS}

In this paper, we present a detailed phenomenological study of the single top (anti)quark production with leptonic decays at the $\mathrm{LHeC}$ at NLO in QCD. The NLO calculations are based on the dipole subtraction method and the complex-mass scheme. We include the full off-shell and nonresonant contributions. The NLO corrections reduce the inclusive cross section by $8.5 \%$, while in a typical fiducial region the NLO corrections reduce the cross section by $14 \%$. We also present predictions of various distributions. The NLO predictions exhibit strong stability under scale variations for both the total cross section and the distributions. The PDF uncertainty can be larger than the scale variations at the NLO, depending on the kinematic region considered.

Moreover, we study the extraction of the top quark mass from measurement of the average transverse momentum of the charged lepton. We find that the statistical error of the extracted top quark mass amounts to $1.1 \mathrm{GeV}$. The theoretical uncertainty due to the scale variations at the NLO are +1.3 and $-0.9 \mathrm{GeV}$. The uncertainties due to input parameters including the $\alpha_{S}$, bottom quark mass, and PDFs are all negligible. In addition, we study the possible improvement on searches of new physics with the NLO predictions of the single top quark production with leptonic decays. We obtain better constraints on $W t b$ effective couplings $g_{R}$ and $\delta V_{L}$ using the NLO predictions than using the LO predictions. The bounds derived using the LO predictions are much weaker when including the scale variations. We anticipate our best bounds on $g_{R}$ and $\delta V_{L}$ at the $95 \%$ C.L. being $[-0.057,0.050]$ and $[-0.010,0.010]$, respectively, as derived using the NLO SM predictions with scale variations.

\section{ACKNOWLEDGMENTS}

The work of J. G. is sponsored by the National Natural Science Foundation of China under Grants No. 11875189 and No. 11835005. M. G. acknowledges valuable conversations with Chuanle Sun, Shurun Yuan, and Shuo Lou. 
[1] J. L. A. Fernandez et al. (LHeC Study Group Collaboration), J. Phys. G 39, 075001 (2012).

[2] P. Agostini et al. (LHeC and FCC-he Study Group Collaborations), arXiv:2007.14491.

[3] H. Mäntysaari (LHeC and FCC-he Study Group Collaborations), Proc. Sci., ICHEP2020 (2021) 553 [arXiv: 2010.05481].

[4] J. Rojo and F. Caola, in Proceedings of the 17th International Workshop on Deep-Inelastic Scattering and Related Subjects, Madrid, 2009 (Science Wise Publishing, Berlin, 2009).

[5] J. L. A. Fernandez et al. (LHeC Study Group Collaboration), arXiv:1211.5102.

[6] A. M. Cooper-Sarkar (LHeC Study Group Collaboration), Proc. Sci., DIS2016 (2016) 274 [arXiv:1605.08579].

[7] H. Paukkunen (LHeC Study Group Collaboration), Proc. Sci., DIS2017 (2018) 109 [arXiv:1709.08342].

[8] A. Caldwell, R. Ent, A. Levy, P. Newman, and F. Olness, arXiv: 1812.08110.

[9] R. A. Khalek, S. Bailey, J. Gao, L. Harland-Lang, and J. Rojo, SciPost Phys. 7, 051 (2019).

[10] H. Abdolmaleki et al. (xFitter Developers Team Collaboration), Proc. Sci., DIS2019 (2019) 025 [arXiv: 1909.00451].

[11] T. Han and B. Mellado, Phys. Rev. D 82, 016009 (2010).

[12] B. Jager, Phys. Rev. D 81, 054018 (2010).

[13] S.S. Biswal, R. M. Godbole, B. Mellado, and S. Raychaudhuri, Phys. Rev. Lett. 109, 261801 (2012).

[14] J. Esteves, J. Phys. Conf. Ser. 645, 012009 (2015).

[15] M. Kumar, X. Ruan, R. Islam, A. S. Cornell, M. Klein, U. Klein, and B. Mellado, Phys. Lett. B 764, 247 (2017).

[16] M. Kumar, X. Ruan, A. S. Cornell, R. Islam, and B. Mellado, J. Phys. Conf. Ser. 623, 012017 (2015).

[17] S. Liu, Y.-L. Tang, C. Zhang, and S.-H. Zhu, Eur. Phys. J. C 77, 457 (2017).

[18] H. Sun, X. Luo, W. Wei, and T. Liu, Phys. Rev. D 96, 095003 (2017).

[19] C. Mosomane, M. Kumar, A. S. Cornell, and B. Mellado, J. Phys. Conf. Ser. 889, 012004 (2017).

[20] O. Flores-Sánchez, J. Hernández-Sánchez, C. G. Honorato, S. Moretti, and S. Rosado-Navarro, Phys. Rev. D 99, 095009 (2019).

[21] H. Hesari, H. Khanpour, and M. M. Najafabadi, Phys. Rev. D 97, 095041 (2018).

[22] L. Delle Rose, O. Fischer, and A. Hammad, Int. J. Mod. Phys. A 34, 1950127 (2019).

[23] C. Han, R. Li, R.-Q. Pan, and K. Wang, Phys. Rev. D 98, 115003 (2018).

[24] R. Li, X.-M. Shen, B.-W. Wang, K. Wang, and G. Zhu, Phys. Rev. D 101, 075036 (2020).

[25] K. Cheung, O. Fischer, Z. S. Wang, and J. Zurita, J. High Energy Phys. 02 (2021) 161.

[26] A. Jueid, J. Kim, S. Lee, and J. Song, Phys. Lett. B 819, 136417 (2021).

[27] S. Forte et al., Eur. Phys. J. C 75, 554 (2015).

[28] R. Li, X.-M. Shen, K. Wang, T. Xu, L. Zhang, and G. Zhu, Phys. Rev. D 97, 075043 (2018).
[29] O. Cakir, A. Senol, and A. T. Tasci, Europhys. Lett. 88, 11002 (2009).

[30] I. T. Cakir, O. Cakir, and S. Sultansoy, Phys. Lett. B 685 , 170 (2010).

[31] S. Dutta, A. Goyal, M. Kumar, and B. Mellado, Eur. Phys. J. C 75, 577 (2015).

[32] I. T. Cakir, A. Senol, and A. T. Tasci, Mod. Phys. Lett. A 29, 1450021 (2014).

[33] I. A. Sarmiento-Alvarado, A. O. Bouzas, and F. Larios, J. Phys. G 42, 085001 (2015).

[34] I. Turk Cakir, A. Yilmaz, H. Denizli, A. Senol, H. Karadeniz, and O. Cakir, Adv. High Energy Phys. 2017, 1 (2017).

[35] H. Sun (LHeC/FCC-eh Top Physics Study Group Collaboration), Proc. Sci., DIS2018 (2018) 186.

[36] C. Schwanenberger, Proc. Sci., EPS-HEP2019 (2020) 635.

[37] W. Liu and H. Sun, Phys. Rev. D 100, 015011 (2019).

[38] B. Yang, B. Hou, H. Zhang, and N. Liu, Phys. Rev. D 99, 095002 (2019).

[39] B. Rezaei and G. R. Boroun, Europhys. Lett. 130, 51002 (2020).

[40] Y.-L. Tang, C. Zhang, and S.-H. Zhu, Phys. Rev. D 94, 011702 (2016).

[41] X.-H. Yang and Z.-J. Yang, arXiv:2103.11412.

[42] M. Czakon, P. Fiedler, and A. Mitov, Phys. Rev. Lett. 110, 252004 (2013).

[43] E. L. Berger, J. Gao, and H. X. Zhu, J. High Energy Phys. 11 (2017) 158.

[44] P. A. Zyla et al. (Particle Data Group Collaboration), Prog. Theor. Exp. Phys. 2020, 083 C01 (2020).

[45] G. Bordes and B. van Eijk, Nucl. Phys. B435, 23 (1995).

[46] R. Pittau, Phys. Lett. B 386, 397 (1996).

[47] T. Stelzer, Z. Sullivan, and S. Willenbrock, Phys. Rev. D 56, 5919 (1997).

[48] T. Stelzer, Z. Sullivan, and S. Willenbrock, Phys. Rev. D 58, 094021 (1998).

[49] B. W. Harris, E. Laenen, L. Phaf, Z. Sullivan, and S. Weinzierl, Int. J. Mod. Phys. A 16, 379 (2001).

[50] B. W. Harris, E. Laenen, L. Phaf, Z. Sullivan, and S. Weinzierl, Phys. Rev. D 66, 054024 (2002).

[51] Z. Sullivan, Phys. Rev. D 70, 114012 (2004).

[52] J. M. Campbell, R. K. Ellis, and F. Tramontano, Phys. Rev. D 70, 094012 (2004).

[53] Z. Sullivan, Phys. Rev. D 72, 094034 (2005).

[54] S. Frixione, E. Laenen, P. Motylinski, and B. R. Webber, J. High Energy Phys. 03 (2006) 092.

[55] Q.-H. Cao, R. Schwienhorst, J. A. Benitez, R. Brock, and C. P. Yuan, Phys. Rev. D 72, 094027 (2005).

[56] S. Alioli, P. Nason, C. Oleari, and E. Re, J. High Energy Phys. 09 (2009) 111; 02 (2010) 011(E).

[57] J. M. Campbell, R. Frederix, F. Maltoni, and F. Tramontano, Phys. Rev. Lett. 102, 182003 (2009).

[58] P. Falgari, P. Mellor, and A. Signer, Phys. Rev. D 82, 054028 (2010).

[59] R. Schwienhorst, C. P. Yuan, C. Mueller, and Q.-H. Cao, Phys. Rev. D 83, 034019 (2011). 
[60] J. Wang, C. S. Li, H. X. Zhu, and J. J. Zhang, arXiv: 1010.4509 .

[61] N. Kidonakis, Phys. Rev. D 83, 091503 (2011).

[62] P. Falgari, F. Giannuzzi, P. Mellor, and A. Signer, Phys. Rev. D 83, 094013 (2011).

[63] R. Frederix, E. Re, and P. Torrielli, J. High Energy Phys. 09 (2012) 130.

[64] J. Wang, C. S. Li, and H. X. Zhu, Phys. Rev. D 87, 034030 (2013).

[65] A. S. Papanastasiou, R. Frederix, S. Frixione, V. Hirschi, and F. Maltoni, Phys. Lett. B 726, 223 (2013).

[66] M. Brucherseifer, F. Caola, and K. Melnikov, Phys. Lett. B 736, 58 (2014).

[67] M. Assadsolimani, P. Kant, B. Tausk, and P. Uwer, Phys. Rev. D 90, 114024 (2014).

[68] P. Kant, O. M. Kind, T. Kintscher, T. Lohse, T. Martini, S. Mölbitz, P. Rieck, and P. Uwer, Comput. Phys. Commun. 191, 74 (2015).

[69] N. Kidonakis, Phys. Rev. D 93, 054022 (2016).

[70] C. Meyer, J. High Energy Phys. 04 (2017) 006.

[71] E. L. Berger, J. Gao, C. P. Yuan, and H. X. Zhu, Phys. Rev. D 94, 071501 (2016).

[72] R. Frederix, S. Frixione, A. S. Papanastasiou, S. Prestel, and P. Torrielli, J. High Energy Phys. 06 (2016) 027.

[73] E. L. Berger, J. Gao, and H. X. Zhu, J. High Energy Phys. 11 (2017) 158.

[74] S. Carrazza, R. Frederix, K. Hamilton, and G. Zanderighi, J. High Energy Phys. 09 (2018) 108.

[75] Z. L. Liu and J. Gao, Phys. Rev. D 98, 071501 (2018).

[76] Q.-H. Cao, P. Sun, B. Yan, C. P. Yuan, and F. Yuan, Phys. Rev. D 98, 054032 (2018).

[77] T. Neumann and Z. E. Sullivan, J. High Energy Phys. 06 (2019) 022.

[78] N. Kidonakis, Phys. Rev. D 99, 074024 (2019).

[79] R. Frederix, D. Pagani, and I. Tsinikos, J. High Energy Phys. 09 (2019) 122.

[80] Q.-H. Cao, P. Sun, B. Yan, C. P. Yuan, and F. Yuan, arXiv:1902.09336.

[81] J. Gao and E. L. Berger, Phys. Lett. B 811, 135886 (2020).

[82] J. Campbell, T. Neumann, and Z. Sullivan, J. High Energy Phys. 02 (2021) 040.

[83] N. U. Basat, Z. Li, and Y. Wang, arXiv:2102.08225 [Phys. Rev. D (to be published)].

[84] T. A. Aaltonen et al. (CDF and D0 Collaborations), Phys. Rev. Lett. 115, 152003 (2015).

[85] A. M. Sirunyan et al. (CMS Collaboration), Phys. Lett. B 808, 135609 (2020).

[86] V. Khachatryan et al. (CMS Collaboration), Phys. Rev. D 93, 072004 (2016).

[87] S. Alekhin, S. Moch, and S. Thier, Phys. Lett. B 763, 341 (2016).

[88] M. Aaboud et al. (ATLAS Collaboration), Eur. Phys. J. C 79, 290 (2019).

[89] H.-J. He and C. P. Yuan, Phys. Rev. Lett. 83, 28 (1999).
[90] T. M. P. Tait and C. P. Yuan, Phys. Rev. D 63, 014018 (2000).

[91] J. J. Liu, C. S. Li, L. L. Yang, and L. G. Jin, Phys. Rev. D 72, 074018 (2005).

[92] J. J. Zhang, C.S. Li, J. Gao, H. Zhang, Z. Li, C. P. Yuan, and T.-C. Yuan, Phys. Rev. Lett. 102, 072001 (2009).

[93] J. Gao, C. S. Li, J. J. Zhang, and H. X. Zhu, Phys. Rev. D 80, 114017 (2009).

[94] J. J. Zhang, C. S. Li, J. Gao, H. X. Zhu, C. P. Yuan, and T.-C. Yuan, Phys. Rev. D 82, 073005 (2010).

[95] J. Wang, C. S. Li, D. Y. Shao, and H. Zhang, Phys. Rev. D 86, 034008 (2012).

[96] Y. Zhang, B. H. Li, C. S. Li, J. Gao, and H. X. Zhu, Phys. Rev. D 83, 094003 (2011).

[97] B. H. Li, Y. Zhang, C. S. Li, J. Gao, and H. X. Zhu, Phys. Rev. D 83, 114049 (2011).

[98] J. Gao, C. S. Li, L. L. Yang, and H. Zhang, Phys. Rev. Lett. 107, 092002 (2011).

[99] Y. Wang, F. P. Huang, C. S. Li, B. H. Li, D. Y. Shao, and J. Wang, Phys. Rev. D 86, 094014 (2012).

[100] G. Cullen, N. Greiner, G. Heinrich, G. Luisoni, P. Mastrolia, G. Ossola, T. Reiter, and F. Tramontano, Eur. Phys. J. C 72, 1889 (2012).

[101] G. Cullen et al., Eur. Phys. J. C 74, 3001 (2014).

[102] S. Catani and M. H. Seymour, Nucl. Phys. B485, 291 (1997); B510, 503(E) (1998).

[103] S. Catani, S. Dittmaier, M. H. Seymour, and Z. Trocsanyi, Nucl. Phys. B627, 189 (2002).

[104] A. Aeppli, G. J. van Oldenborgh, and D. Wyler, Nucl. Phys. B428, 126 (1994).

[105] A. Denner, S. Dittmaier, M. Roth, and D. Wackeroth, Nucl. Phys. B560, 33 (1999).

[106] T. Hahn, Comput. Phys. Commun. 207, 341 (2016).

[107] T.-J. Hou et al., Phys. Rev. D 103, 014013 (2021).

[108] M. Cacciari, G. P. Salam, and G. Soyez, J. High Energy Phys. 04 (2008) 063.

[109] H. Esch (ATLAS Collaboration), arXiv:1411.3879.

[110] A. M. Sirunyan et al. (CMS Collaboration), Eur. Phys. J. C 77, 354 (2017).

[111] A. Czarnecki and M. Jezabek, Nucl. Phys. B427, 3 (1994).

[112] B. Aubert et al. (BABAR Collaboration), Phys. Rev. Lett. 97, 112001 (2006).

[113] S. R. Yuan, M. S. Gao, and J. Gao, J. High Energy Phys. 04 (2021) 054.

[114] L. A. Harland-Lang, A. D. Martin, P. Motylinski, and R. S. Thorne, Eur. Phys. J. C 75, 204 (2015).

[115] A. Buckley, J. Ferrando, S. Lloyd, K. Nordström, B. Page, M. Rüfenacht, M. Schönherr, and G. Watt, Eur. Phys. J. C 75, 132 (2015).

[116] L. A. Harland-Lang, A. D. Martin, P. Motylinski, and R. S. Thorne, Eur. Phys. J. C 76, 10 (2016).

[117] P. Azzi et al., CERN Yellow Rep. Monogr. 7, 1 (2019).

[118] F. Bach and T. Ohl, Phys. Rev. D 86, 114026 (2012).

[119] B. Grzadkowski, M. Iskrzynski, M. Misiak, and J. Rosiek, J. High Energy Phys. 10 (2010) 085. 
[120] J. A. Aguilar-Saavedra, Nucl. Phys. B843, 638 (2011); B851, 443(E) (2011).

[121] J. A. Aguilar-Saavedra, Nucl. Phys. B812, 181 (2009).

[122] J. Alwall, R. Frederix, S. Frixione, V. Hirschi, F. Maltoni, O. Mattelaer, H. S. Shao, T. Stelzer, P. Torrielli, and M. Zaro, J. High Energy Phys. 07 (2014) 079.
[123] E. Conte, B. Fuks, and G. Serret, Comput. Phys. Commun. 184, 222 (2013).

[124] C. Degrande, G. Durieux, F. Maltoni, K. Mimasu, E. Vryonidou, and C. Zhang, Phys. Rev. D 103, 096024 (2021). 